\title{
Schmerztherapie
}

\section{Wird jetzt noch mehr an Opioiden gespart?}

- Bei der Versorgung von Schmerzpatienten stellen Schmerztherapeuten seit Jahren eklatante Mängel fest. Beklagt werden sowohl strukturelle Defizite (zu wenig spezialisierte Schmerzzentren) als auch eine Unterversorgung mit geeigneten Analgetika. Die vom Gesetzgeber geplanten Einsparungen im Arzneimittelsektor schüren nun zusätzliche Ängste.

Mittels Zwangsrabatten und anderen Kostendämpfungsinstrumenten (Großhandelszuschlag, Apothekenrabatt etc.) plant der Gesetzgeber allein in Sachen Arzneimittelausgaben kurzfristig wirksame Entlastungen der gesetzlichen Krankenversicherung (GKV) in Höhe von 2,2 Mrd. Euro nach Einschätzung von Ulrich Dietz, beim BMG Leiter des Referats Arzneimittelversorgung, ein durchaus realistisches Volu- men. Am Sparen beteiligen sollen sich auch all jene Ärzte, die BtMVV-pflichtige hochpotente Opioide verordnen, z. B. durch die in der Arzneimittelvereinbarung 2011 vorgesehenen Verordnungsanteile. So soll etwa der Anteil von generischem Morphin am Gesamtvolumen der Opioide mindestens $40 \%$ betragen und der Anteil der transdermalen Opioide an allen Opioiden höchstens $50 \%$.

\section{Bioverfügbarkeit generischer Opioide kann schwanken}

Dass es mit generischen Opioiden jedoch so seine Tücken haben kann, machte Prof. Dr. Jürgen Fritze vom Verband der privaten Krankenversicherung e.V., Köln, deutlich. Weil sich die Bioverfügbarkeit generischer Opioide im Vergleich mit dem Originalprä- parat in einem Bereich zwischen 80 und $125 \%$ bewegen dürfe, berge dies ein substanzielles Risiko sowohl von Unterdosierungen als auch von Intoxikationen.

Mit dem Gesetz zur Neuordnung des Arzneimittelmarktes (AMNOG) wird es für forschende pharmazeutische Unternehmen künftig nicht leichter, innovative Medikamente zu einem adäquaten Preis auf den Markt zu bringen. Um nicht auf einen Festbetrag eingestuft zu werden, bedarf es neben der Zulassung des Nachweises eines Zusatznutzens gegenüber Medikamenten, die schon auf dem Markt sind.

- Dr. Ludger Riem

Quelle: Aachener Workshop "Zukunft der Schmerztherapie“, 3./4. Dezember 2010 (Veranstalter: Grünenthal)

\section{Non-Ergot-Dopaminagonist in Retardformulierung Parkinsontherapie einfach gemacht}

- Bei neu diagnostizierten Parkinsonpatienten unter 70 Jahren und ohne wesentliche Komorbiditäten empfehlen die Leitlinien der Deutschen Gesellschaft für Neurologie (DGN) eine Monotherapie mit NonErgot-Dopaminagonisten.

Gerade bei Patienten im Frühstadium der Parkinsonerkrankung, die motorisch wenig beeinträchtigt und häufig berufstätig sind, sei ein möglichst einfaches Therapieregime anzustreben, erklärte Prof. Dr. Wolfgang Jost, Wiesbaden. Dazu bieten sich lang wirksame Formulierungen an wie retardiertes Pramipexol (Sifrol ${ }^{\circledR}$ Retard), das bis zu einer Höchstdosis von 3,15 mg als tägliche Einmalgabe verabreicht werden kann. Die Einmalgabe trage erheblich zu einer besseren Compliance bei, so Jost.

\section{Wirkstoff wird über den Tag gleichmäßig freigesetzt}

Der Non-Ergot-Dopaminagonist verbesserte in einer Studie den UPDRS-II- und -IIIGesamtscore über 18 Wochen im Vergleich

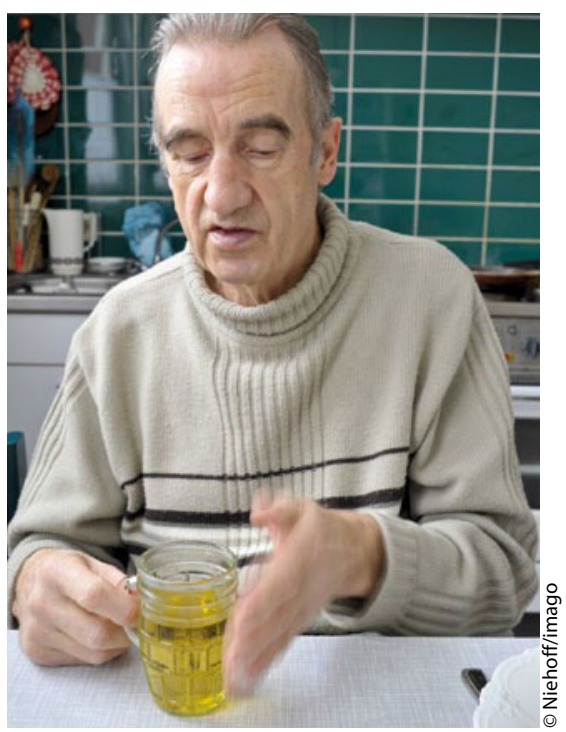

Auch bei Parkison: Je einfacher die Therapie, desto besser die Compliance.

zu Placebo um durchschnittlich 8,1 Punkte (Hauser et al. Mov Disord 2010). Die Retardformulierung setzt den Wirkstoff gleichmäßig über den Tag frei - ohne Fluktuationen, wie sie unter täglicher Mehrfachgabe auftreten.

Die Neueinstellung beginnt mit 0,26 $\mathrm{mg}$ in der ersten Woche und wird in der zweiten Woche auf 0,52 mg und auf 1,05 $\mathrm{mg}$ in der dritten Woche erhöht. Werden im Verlauf weitere Anpassungen erforderlich, kann die Dosis in wöchentlichen Abständen um 0,52 mg bis zur Höchstdosis von 3,15 mg mit einer Tablette täglich gesteigert werden.

„Die Umstellung von der mehrmals täglichen Applikation von Pramipexol auf die Retardtablette kann problemlos über Nacht erfolgen", sagte Jost. Eine Umstellungsstudie mit 155 Patienten zeigte, dass die Wirksamkeit und Verträglichkeit beider Formulierungen vergleichbar sind.

- Michael Koczorek

Quelle: Presse-Round-Table „Basis einer modernen Parkinsontherapie: Sifrol ${ }^{\oplus}$ Retard “, Ingelheim, 3. Februar 2011 (Veranstalter: Boehringer Ingelheim) 